\title{
The Outer Detector (OD) system for the Hyper-Kamiokande experiment
}

\author{
Stephane Zsoldos ${ }^{a, b, c, *}$ \\ ${ }^{a}$ Department of Physics, King's College London, \\ Strand, London WC2R 2LS, United Kingdom \\ ${ }^{b}$ Department of Physics, University of California, Berkeley, \\ CA 94720, Berkeley, USA \\ ${ }^{c}$ Lawrence Berkeley National Laboratory, \\ 1 Cyclotron Road, Berkeley, CA 94720-8153, USA \\ E-mail: stephane.zsoldos@kcl.ac.uk, zsoldos@berkeley.edu
}

Hyper-Kamiokande, scheduled to begin construction as soon as 2020, is a next generation underground water Cherenkov detector, based on the highly successful Super-Kamiokande experiment. It will serve as a far detector, $295 \mathrm{~km}$ away, of a long baseline neutrino experiment for the upgraded J-PARC beam in Japan. It will also be a detector capable of observing — far beyond the sensitivity of the Super-Kamiokande detector - proton decay, atmospheric neutrinos, and neutrinos from astronomical sources.

An Outer Detector (OD) consisting of PMTs mounted behind the inner detector PMTs and facing outwards to view the outer shell of the cylindrical tank, would provide topological information to identify interactions originating from particles outside the inner detector. Any optimization would lead to a significant improvement for the physics goals of the experiment, which are the measurement of the CP leptonic phase and the determination of the neutrino mass hierarchy.

An innovative new setup using small 3" PMTs is being proposed for the Hyper-Kamiokande OD. They would give better redundancy, spatial, and angular resolution, as there would be twice or three times more photosensors that the original 8" design proposal of the experiment, and for a reduced cost. Several 3" PMT candidates considered for the Hyper-Kamiokande OD have been characterized for this study. They all show a very low dark counts and good collection efficiency, which makes them excellent choice to be used in the experiment.

40th International Conference on High Energy physics - ICHEP2020

July 28 - August 6, 2020

Prague, Czech Republic (virtual meeting)

${ }^{*}$ Speaker 


\section{Introduction}

Hyper-Kamiokande [1] (Hyper-K) is the successor of the Super-Kamiokande [2] (Super-K) experiment which was awarded with the Nobel Prize in 2015 for the joint discovery with the SNO [3] experiment of atmospheric neutrino oscillations. This new experiment will consists of two cylindrical water tanks that are $72 \mathrm{~m}$ in height and $68 \mathrm{~m}$ in diameter, for a total volume up to $0.5 \mathrm{Mm}^{3}$ per tank.

Hyper-K will be a multipurpose neutrino detector with a rich physics program that aims to address some of the most significant questions facing particle physicists today. Oscillation studies from accelerator, atmospheric and solar neutrinos will refine the neutrino mixing angles and mass squared difference parameters and will aim to make the first observation of asymmetries in neutrino and antineutrino oscillations arising from a CP-violating phase, shedding light on one of the most promising explanations for the matter-antimatter asymmetry in the Universe. The search for nucleon decays will probe one of the key tenets of Grand Unified Theories. In the case of a nearby supernova, Hyper-K will observe an unprecedented number of neutrino events, providing much needed experimental data to researchers seeking to understand the mechanism of the star explosion. Finally, the detection of astrophysical neutrinos from sources such as dark matter annihilation, gamma ray burst jets, and pulsar winds could further our understanding of some of the most spectacular, and least understood, phenomena in the Universe.

Hyper-K detectors consist of a cylindrical tank filled with a quarter million tons of water. Facing inwards arranged against the inner wall of the cylinder will be hundreds of thousands of Photo Multiplier Tubes (PMTs) capable of detecting the faint signatures of neutrinos interacting with water (the Inner Detector, ID).

An Outer Detector (OD) consisting of PMTs mounted behind the ID PMTs and facing outwards to view the outer shell of the cylindrical tank, would provide topological information to identify interactions originating from particles outside the ID. The OD is an essential element of Hyper-K that will serve as a highly granular instrument for identifying and removing background events.

\section{Mass hierarchy sensitivity}

The determination of the neutrino mass hierarchy is one of Hyper-K's main goals, in concert with the leptonic $\mathrm{CP}$ phase $\delta_{\mathrm{CP}}$ measurement, which can be done using accelerator or atmospheric neutrinos. For the latter, they provide excellent sensitivity to many of the remaining open questions in oscillation physics.

As atmospheric neutrinos span both low and high energies as well as long and short path lengths, they are in principal sensitive to all parameters to test the PMNS matrix unitarity. That being said, the most apparent oscillation features are driven by the so-called atmospheric mixing parameters, $\theta_{23}$ and $\Delta m_{32}^{2}$, and they induce a deficit of observed upward-going $v_{\mu}$ interactions at predominantly multi-GeV energies as these neutrinos oscillate into primarily unobserved $v_{\tau}$. Matter-induced parametric oscillations [4] [5] in the energy range between 2 to $10 \mathrm{GeV}$ lead to significant enhancement of the appearance probability for upward-going neutrinos depending upon the mass hierarchy. For the normal (inverted) hierarchy neutrino (antineutrino) oscillations are enhanced. This enhancement leads to appearance probabilities around $50 \%$ for both hierarchies. 
The separation of atmospheric neutrino data into neutrino-like and antineutrino-like subsets can therefore be used to extract the hierarchy signal.

There are three basic event topologies used in the atmospheric neutrino analysis which cover different neutrino energies. The fully-contained (FC) sample includes particles with vertices inside the fiducial volume which stop before leaving the ID. It is the lowest-energy sample ranging from a few hundred $\mathrm{MeV}$ up to about ten $\mathrm{GeV}$. The partially-contained (PC) sample contains events that have vertices in the fiducial volume, but produce leptons that leave the ID. They have long tracks and so are almost exclusively from $v_{\mu}$ interactions and range in energy from a few $\mathrm{GeV}$ up to tens of $\mathrm{GeV}$. These events have better direction resolution than FC events due to their higher energy, but worse energy resolution since the exiting muon carries some energy out of the detector. Upward-going muon events contain muons that start in the surrounding rock and then enter and pass through the OD into the ID. This sub-sample also starts at a few $\mathrm{GeV}$ but extends up to hundreds of TeV. These events are only included if they are up-going, where the bulk of the Earth has shielded the detector from the otherwise overwhelming cosmic-ray muons.

Increasing the mass hierarchy sensitivity for Hyper-K is possible by working on two research axes: First of all, since the analysis is based on the electron appearance in atmospheric neutrino samples, any substantial improvement expanding the fiducial volume of the detector would lead to an increase in statistics and therefore a better sensitivity. This can be tackled both using software and hardware developments. Recently, Super-K has published results using a new reconstruction software called fiTQun [6] now able to reconstruct up to 6 Cherenkov rings produced by electron, muon, or pion particle hypotheses. This allowed to increase the volume accessible to the analysis by $32 \%$ [7]. Hyper-K will obviously use this reconstruction algorithm, and more developments are also expected thanks to research in the area of Machine Learning, for example.

Secondly, we identified that the biggest systematic error contribution comes from the energy scale calibration, which is about 1 to $2 \%$ depending on Super-K runs. The analysis select events with reconstructed energy between 2 and $10 \mathrm{GeV}$. Calibration at this level is performed using sidebands from $\mathrm{GeV}$ stopping muons. Therefore any improvements on the sensitivity to PC events, and better calorimetry by reconstructing exiting events, could also lead to a sensible improvements to the mass hierarchy sensitivity.

To address both of these issues, we proposed a novel design for the Hyper-K OD, using a large area of small 3" PMTs. An increased number of PMTs even at the expenses of a smaller area covered by the photo-cathodes allow for a better signal redundancy, as well as better spatial and angular resolutions.

\section{OD Photosensors}

Building on top of the experience acquired while operating Super-K, several criteria have been laid down to select the best photosensors candidates. The OD is a veto for background particles, based on cluster of PMTs hits above a certain threshold, defined by the PMTs dark counts. Therefore, the OD segment is used as a photon counter, compared to the ID where the event topology is also used to reconstruct the neutrino energy and vertex position. In the OD case, the amount of information contains in an event increase linearly with the number of PMTs, as defined by the information entropy of our signal, $H=-\log _{2} 2^{-N}$, where $N$ is the Number of PMTs. 
Hence, three criteria emerge for building an efficient OD for Hyper-K: we have to increase the number of PMTs, achieved by using smaller and inexpensive 3" PMTs with a small dark rate to lower the trigger threshold and of course a good light collection, to set an efficient trigger to background events.

In light of this, several PMTs candidates have been selected to be characterised : two Hamamatsu PMTs, the 3" R14374 and the 3.5" R14689; one Electron Tubes 3.5" PMT model ET9320KFL; and one HZC 3.5" PMT model XP82B20. These PMTs are shown in Fig.1. The 3" R14374 Hamamatsu is the same PMT used in the Km3NeT experiment[8], the other 3.5" candidates are in development and in need of accurate measurements from various sources.
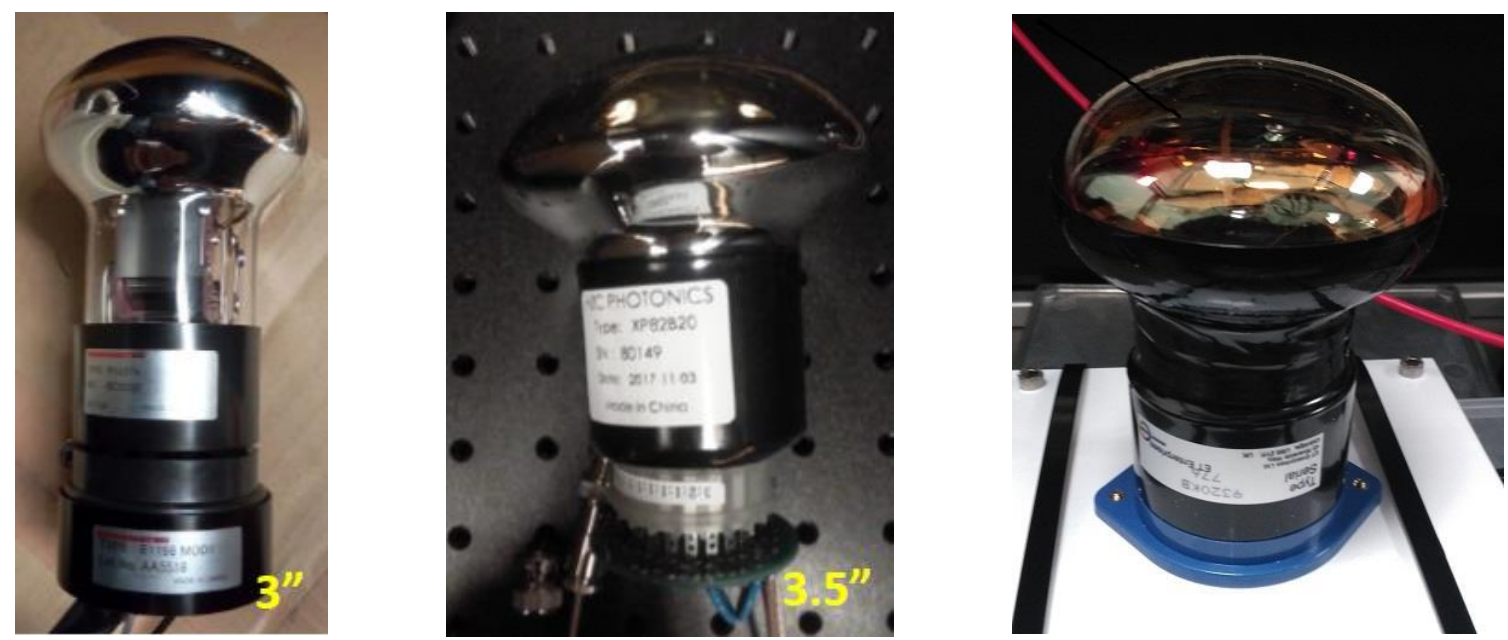

Figure 1: Left : Hamamatsu 3" R14374. Center : HZC 3.5" XP82B20. Right : ETEL 3.5" ET9320KFL.

These small PMTs have also the advantage of a reduced dark rate, measured to be at the hundreds of kHz, one order of magnitude below typical 8" PMTs [9]. Hence, the contribution to dark coincidence will be mitigated by both a lower dark count and using an higher number of hits.

\section{Light collection enhancement system}

The Hyper-K OD will also adopt a similar strategy as its predecessor Super-K and use wavelength-shifting (WLS) plastic plates around the PMTs photocathode in order to increase the light collection. The goal would be to trap any Cerenkov photons, whom spectrum is mostly in the UV region, who would have been missed by the PMT and re-emit it at a greater wavelength to match the PMT quantum efficiency (QE) peak. The plastic, usually Polyvinyl Toluene (PVT) as it is easy to load with anthracene or any other dopant, has a refraction index $n_{\mathrm{WLS}}=1.57$, which is higher than water, typically in Hyper-K at $10^{\circ} \mathrm{C}\left(n_{\text {Water }}=1.33\right)$, and therefore total reflection can be achieved inside the plate. The collection probability $P_{\mathrm{CE}}$ is the ratio between the solid angle defined by the total reflection angle $\theta_{\text {crit }}=\sin ^{-1} \frac{n_{\text {Water }}}{n_{\mathrm{WLS}}} \approx 1 \mathrm{rad}$, and the total solid angle of re-emission:

$$
P_{\mathrm{CE}}=1-\frac{2 \pi\left(1-\cos \theta_{\text {crit }}\right)}{2 \pi}=\cos \theta_{\text {crit }} \approx \cos (1 \mathrm{rad})=0.54
$$

Hence, $54 \%$ of the light re-emitted in this particular case will be trapped inside the plate. Since the refractive index of the water is fixed, the value of this figure increases only with the plastic 
refractive index. Fig. 2 shows this system as it is deployed in Super-K, using a 8" PMT and a $60 \times 60 \mathrm{~cm}^{2}$ WLS plate. A UV lamp illuminates the plate as the control that the light is being guided as intended.

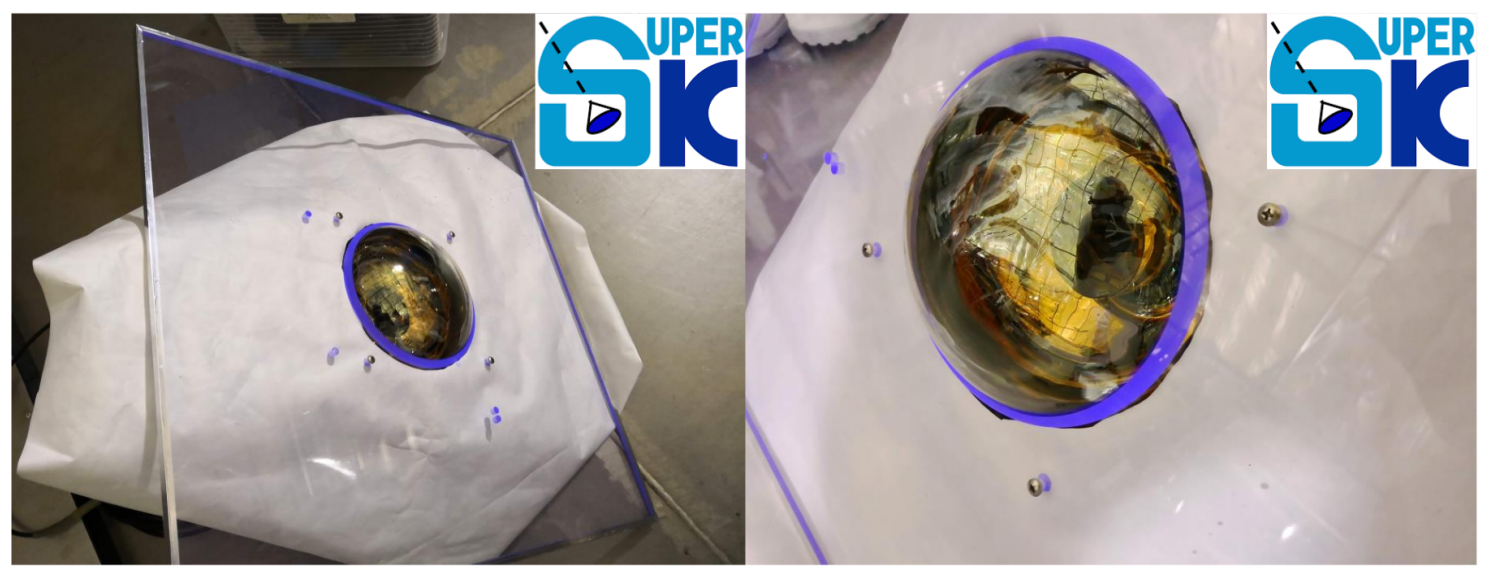

Figure 2: The Super-K OD PMTs coupled to a WLS plate system.

Several WLS plates are being considered for the Hyper-K OD: existing models from Eljen EJ-286 based on PVT, and prototypes being developed in close collaboration with Kuraray and LabLogic companies in Japan and in the UK respectively. These prototypes use PMMA which has a lower index of refraction $n_{\text {PMMA }}=1.49$. Fig. 3 shows the absorption and emission spectra measured at U. of Edinburgh, using a UV spectrometer. The best candidate should have the highest absorption in the UV regime, where the maximum Cherenkov photons are emitted, and re-emit at the maximum of the PMT QE.
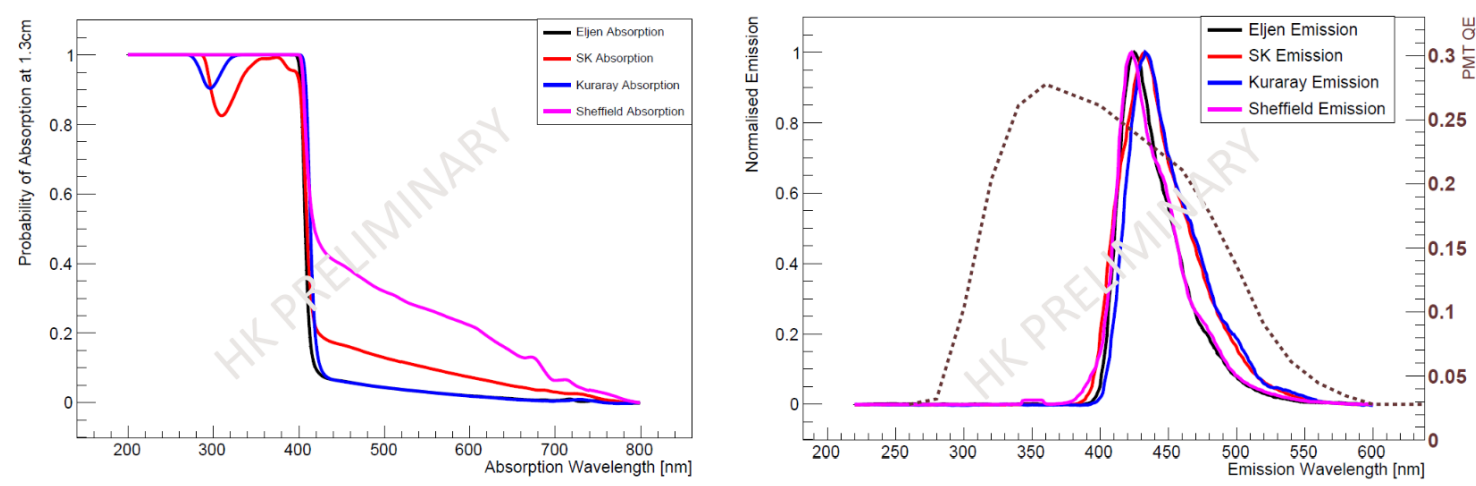

Figure 3: Left: absorption measured for each plate using a $1.3 \mathrm{~cm}$ sample with respect to the wavelength of the light. Right: emission spectrum from each plate, and with the Hamamatsu R14374 PMT QE overlaid.

In order to evaluate the light collection enhancement factor one can expect using the WLS plate coupled to the PMT, a setup has been deployed at U. of Edinburgh where a LED diode is flashed at different position with respect to the center of the PMT. The efficiency is then normalized to the fraction of direct light collected by the PMT when the LED is right above the center of the photocathode, in the absence of the plate. Fig. 4 (left) shows the setup with a 3" ETEL 9320KFL 
PMT inside a dark box and coupled at a $24 \times 24 \mathrm{~cm}^{2}$ EJ-286 WLS plate. The LED light is guided with an optical fiber moved at several position from the center. Two cases have been considered, without and with an additional cladding made of mylar on the side of the plate, in order to reflect any light exiting the plate from the edge.
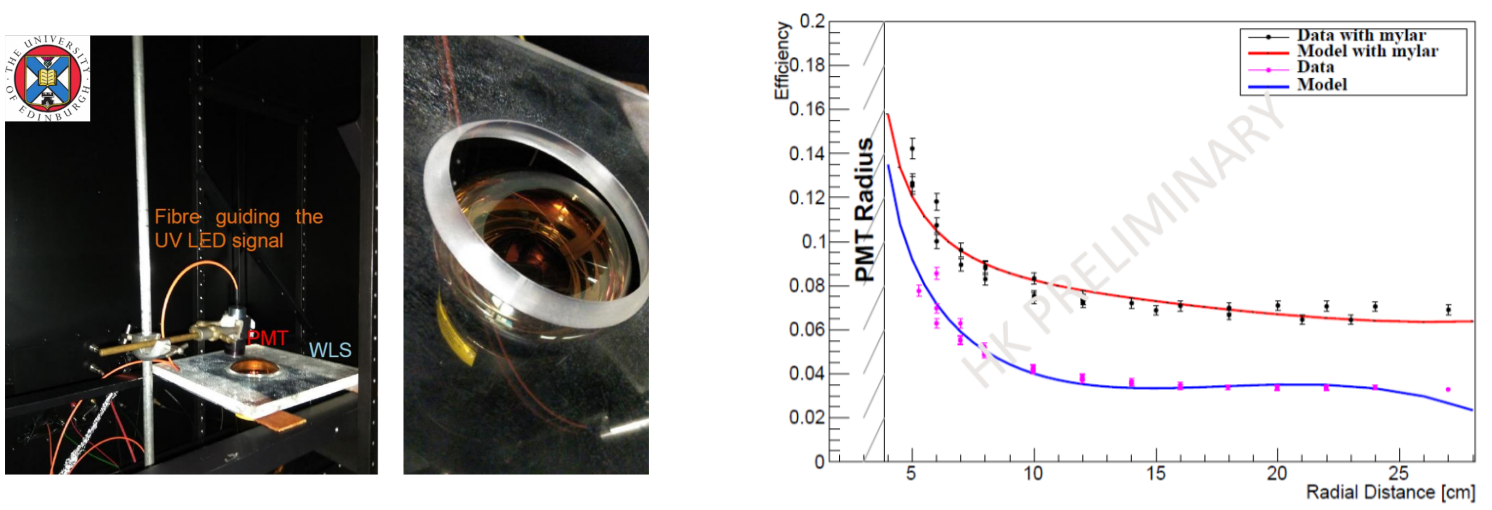

Figure 4: Left: 3" ETEL 9320KFL and EJ-286 WLS plate coupled inside a dark box at U. of Edinburgh. Right: additional light collected with the WLS plate w.r.t to the LED position from the center of the PMT photocathode.

The results are shown in Fig. 4 (right). The addition of the mylar cladding covering the edges is essential as it increases the collected light by a factor 2 . An analytical model has been developed in order to fit the data without and with cladding, and it is represented overlaid on the data points. Thanks to this model, we can integrate the efficiency measured on the area of the plate, which leads to an expected total increase of light collected of 2.6 for an expected $50 \times 50 \mathrm{~cm}^{2}$ plate used inside the Hyper-K OD.

\section{References}

[1] K. Abe et al. [Hyper-Kamiokande Collaboration], arXiv:1805.04163 [physics.ins-det].

[2] Y. Fukuda et al. [Super-Kamiokande Collaboration], Nucl. Instrum. Meth. A 501, 418 (2003).

[3] J. Boger et al. [SNO Collaboration], Nucl. Instrum. Meth. A 449 (2000) 172

[4] S. P. Mikheev and A. Yu. Smirnov, Sov. J. Nucl. Phys. 42, 913 (1985),

[5] L. Wolfenstein, Phys. Rev. D 17, 2369 (1978).

[6] A. D. Missert [T2K Collaboration], J. Phys. Conf. Ser. 888 (2017) no.1, 012066.

[7] M. Jiang et al. [Super-Kamiokande Collaboration], PTEP 2019 (2019) no.5, 053 F01

[8] S. Aiello et al. [KM3NeT Collaboration], JINST 13, no. 05, P05035 (2018).

[9] S. Zsoldos, arXiv:1908.07864 [physics.ins-det]. 